\title{
Parallel-kinematics XYZ MEMS Part 1: Kinematics and Design for Fabrication
}

\author{
Jorge Correa, Bonjin Koo, and Placid Ferreira*
}

Department of Mechanical Science and Engineering, 1206 West Green Street, MC-244, University of Illinois at Urbana-Champaign, Urbana, IL 61801, USA

\begin{abstract}
Micro- and nanopositioning systems are widely used in the field of nanotechnology for probing, imaging, and increasingly for processing. This two-part set of papers presents a MEMS-scale parallel-kinematics mechanism, designed to achieve pure spatial (X, Y and Z) translation. With three independent kinematic chains connecting the end-effector to the base, a fully functional mechanism with axis actuation and displacement sensing is realized in a double device layer ("oreo") SOI wafer using only conventional, microfabrication processes. This paper, the first in a two paper set, presents the mechanism, specially designed for scalable microfabrication. It analyzes its kinematics and dynamics, and characterizes its workspace. Part II of this set of papers describes the detailed design, fabrication, characterization and control of the devices.
\end{abstract}

Keywords: XYZ MEMS; parallel-kinematics mechanisms; nanopositioning; flexure stage;

\section{Introduction}

Ilicon-based micro-electro-mechanical positioning systems find application in the fields of micro- and nanomanipulation and nanoprobe technology. With their ability to regulate displacements and forces with high resolution, their applications include switches [1], micro force sensors [2], [3] [4], data storage devices, scanning probe microscopy actuators [5], [6], micro optical lens scanners and aligners [7], [8], [9], [10]. Some of the important reasons why MEMS positioners play an important role in nanotechnology are: size, high dynamic range, high resolution of motion or force, and integrated fabrication with other elements of micro-systems. With the latter often being critical consideration in applications that embed MEMS-based nanopositioners, while piezoelectric actuators [11], shape memory alloy actuators [12], electromagnetic actuators, electrothermal actuators [13] have been suggested, electrostatic comb-drive actuators [1]- [10], [14], [15], [16], [17], [18] are most commonly used.

Complex bulk microfabricated devices with 3D features are challenging to produce. Most MEMS devices therefore remain simple in shape due to the two-dimensional (2D) and two and a half dimensional (2.5D) nature of most of the common microfabrication processes such as deep reactive ion etching (DRIE) and (LIGA. For this reason, many of the MEMS-scale mechanisms are flat structures built on a device layer of an SOI substrate, anchored to the buried oxide (BOX) layer, partially removed to create a suspended device layer structure [19]. Among the recent efforts to produce XYZ nanopositioning MEMS stages is the fabrication of a 3-axis positioning stage with comb drives held by tethering beams [20]. The combs are used to move the center stage in $\mathrm{X}$ and $\mathrm{Y}$ direction and a parallel plate electrostatic actuator is used to position the center stage vertically. Initially, the mechanism has decoupled XYZ motions and low actuation voltage is required (as the actuators are lined up with the motion directions). Although integrating dedicated gap-closing type actuators is effective in achieving independent out-of-plane motion, this type of a scheme requires high actuation voltages and suffers from pull-in instabilities, limiting the range of motion and creating difficulties when the end-effector of the machine may pick up external disturbing forces. A 3-axis nanopositioning stage using electrostatic actuation is reported in [21]. In this work, the out-of-plane motion is obtained by the fabrication of two sets of comb actuators with inclined orthogonal suspensions on a SOI substrate by means of inductively coupled plasma (ICP) etching and focused ion beam (FIB) machining The stage has a reported workspace of less than $0.5 \mu \mathrm{m}$ at a $100 \mathrm{~V}$ of actuation in XYZ direction. Alternatively, vertical comb-actuators have been used to generate motion in the out-of-plane direction and they have been found useful in applications such as scanning micro-mirrors [22]. An example of a serial mechanism for 3-axis micro motion stage is given in [23]. This design assembles a nested structure of electrostatically driven stages to produce the XY motion and an electro-thermally driven stage to produce the $\mathrm{Z}$ motion. The stage relies on a buckling phenomenon to produce vertical actuation. The range of motion of the stage is reported to be 53.98, 49.15 and $22.91 \mu \mathrm{m}$ along X, Y and $\mathrm{Z}$ respectively. Electrical isolation together with signal and power routing are challenging in such designs. Further, error characterization and compensation are required to reduce the effects of coupling between the individual stages. Dong and Ferreira [24] demonstrated controlled XYZ motion at a cantilever tip by actuating a cantilever mounted on an XY stage with a tilt-plate electrostatic actuator. Here the out-of-plane motion along the z-axis was limited to around 2 microns.

Parallel-kinematics mechanisms, that have been increasingly studied for use in macro and meso-scale positioning systems [11], [24], [26] can be designed so as to be better suited for silicon-based, MEMS-scale micro- and nanopositioners. A parallelkinematics mechanism (PKM) consists of a fixed base and movable end-effector connected in parallel by multiple independent

* Corresponding author. E-mail: pferreir@illinois.edu). 
kinematic chains. Normally, the degrees-of-freedom (DOF) at the end-effector is determined by the number of independent kinematic chains. Each kinematic chain actuates the end-effector in one direction and accommodates or admits displacements along the other DOF of the end-effector. Together, all the chains restrict the undesired motions (for example, rotations for a translational stage). In this way, a mechanism is realized in which the desired DOF are spanned by the actuators and the unwanted DOF are restricted by the interaction of the kinematic chains. Parallel-kinematics mechanisms generally produce high structural stiffness because of their truss-like structures, resulting in fast response times. Furthermore, if appropriately designed, PKMs can result in configurations where near complete decoupling of the actuation is achieved. PKMs are criticized for small workspaces because the motion range of a PKM is restricted to the intersection of the motion range of all its kinematic chains. This is a valid criticism for macroscale systems, where the kinematic joints and actuators have large permissible motion ranges. However, for stages built by MEMS processes, the motion range of the stage is more likely to be governed by the limits of the actuators and flexure joints than the mechanism itself. Reference [25] introduces a XYZ parallel-kinematics micromanipulator composed of a circular positioning platform with three legs equally space around it. Each of the leg is composed of a slider mechanism and a parallel-kinematics mechanism. This design was shown to produce out-of-plane displacements greater than 250 $\mu \mathrm{m}$ when displacements of $45 \mu \mathrm{m}$ were input by microprobes at each of the sliders. The mechanisms were fabricated using the MUMPs and SUMMIT process, required several (at least 7 structural and sacrificial) layers and lacked actuation and sensing. The devices had to be externally actuated with microprobes to verify and study their motions.

In this paper, a parallel-kinematics scheme, particularly well-suited for a flexure-based SOI MEMS stage with XYZ motion capabilities is presented and analyzed for its kinematics and dynamics. The stage has three independent kinematic chains and is designed to be operated by linear comb actuators. The kinematic chains make in-parallel mechanical connections between the base of the manipulator to its end effector. Each kinematic chain has one actuated prismatic joint, a parallelogram 4-bar mechanism and an out-of-plain link. The end-effector is held by the out-of-plane links, and each of the chains is located to have $120^{\circ}$ rotational symmetry in the XY plane. The device is designed for fabrication on double device layer ('oreo'), silicon-oninsulator (SOI) substrate, consisting of two device layers and a handle layer sandwiched between them, and insulated from them by buried oxide (BOX) layers. The high aspect ratio structures such as the integrated comb drives and out-of-plane links are achieved by deep reacting ion etching (DRIE). Both handle layer and the device layers are used in the fabrication process to produce the three-dimensional spatial features of the kinematic structure. The 4-bar mechanism structure and the comb drives are mapped onto the device layer 1(DL1) and connected to the out-of-plane link by out-of-plane hinges. The end-effector and a set of out-of-plane hinges are mapped to the device layer 2 (DL2) and are also connected out-of-plane link. The out-of-plane link spans all the layers, DL1, DL2, handle and BOX of the substrate, connecting the components patterned into the two layers, DL1 and DL2. Figure 1 shows a schematic of the XYZ MEMS kinematics structure and how this structure is mapped onto the layers of the SOI substrate.

This paper, the first in a 2-part series, will focus on developing the theory (kinematics, dynamics, workspace and forces) required to design and control this mechanism. It gives examples, using representative values (obtained from the detailed design of a device in the second paper), of how the formulae developed characterize these important aspect of a designed device. The second paper in this series will focus on the detailed design, fabrication, characterization and control of an XYZ MEMS positioner. Besides their contributions to the theory, analysis, design and integration of a novel out-of-plane MEMS positioner with previously unrealized capabilities, this set of papers also demonstrates that, just like conventional scale positioning systems, multi-purpose, self-contained MEM-scale positioning stages can be realized with traditional fabrication processes. Further, together these two papers provide the complete design methodology and fabrication steps for designing and realizing such positioning systems.

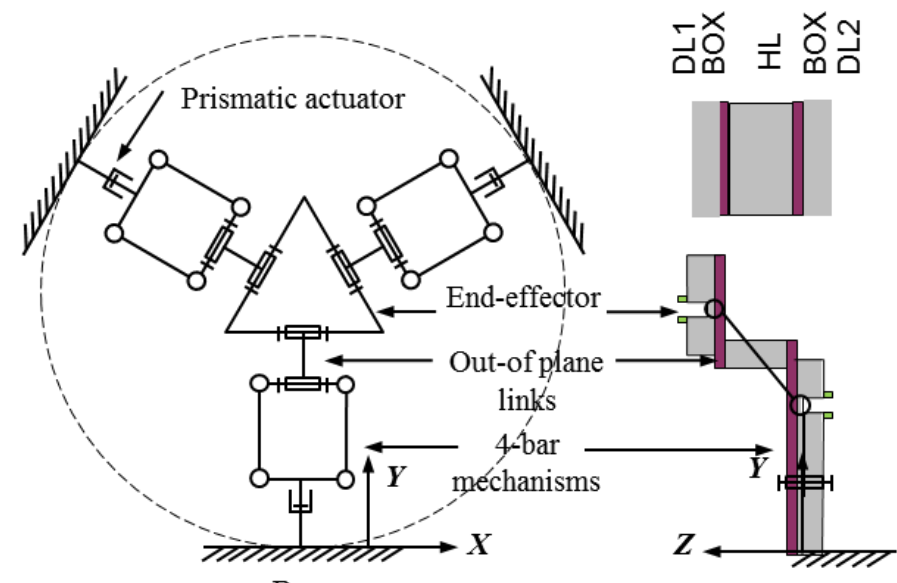

Base 


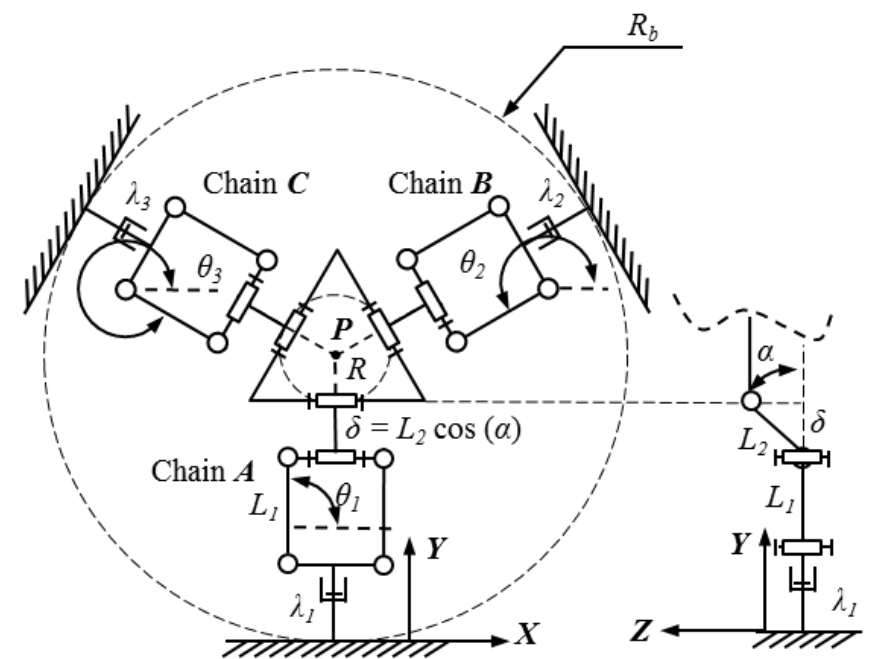

Fig. 2.Kinematics parameters, variables and frame location of the XYZ stage.

\section{Schematic design and kinematic analysis of the PK-XYZ-MEMS stage}

The proposed scheme for the Parallel Kinematics XYZ MEMS (PK-XYZ-MEMS) stage is an adaptation of the Parallel Kinematics XYZ Nano-Positioning stage (PKXYZNP) [26]. Like the PKXYZNP, it has three independent kinematic chains, but with the rotational actuators replaced by comb-drive actuators and the motion of the second four-bar mechanism decoupled into an in-plain four-bar mechanism and an out-of-plane link. These modifications allow for miniaturization of the stage and its implementation as a MEMS device. As previously mentioned, and shown schematically in Figure 2, the kinematic structure of each chain consist of an in-plane (here the XY plane) parallelogram four-bar mechanism whose base is attached to an in-plane prismatic actuator. The connector of the 4-bar is connected to a rigid out-of-plain link by a revolute joint whose axis is in the XY plane, thus permitting the link to swing out of the XY plane. This other end of this link is connected to the end-effector by a revolute joint. Each chain is actuated by an in-plane prismatic actuator that moves along a fixed line in the XY plane. The parallelogram four-bar mechanism of each chain is designed so that its connector undergoes pure translation along a circular path in the XY plane and its orientation is always parallel to its base. The only degree of freedom allowed by the out-of-plane link is a rotation around an axis, also parallel to the base of the four-bar mechanism, which never changes orientation relative to the base of the mechanism. Therefore, the edge connecting the end-effector to the out-of-plane link never changes orientation. Since we have 3 kinematic chains, we have more than two different, non-parallel lines on the end-effector that always maintain the same orientation, regardless the configuration of the stage. This explains why the table experiences only translation. Referring to Figure 2, the four-bar parallelograms span the XY plane while the out-of-plane links allow the out-of-plane motion of the stage. Fixing the position of the three prismatic actuators, results in fixing the end-effector at a XYZ location.

\section{A. Forward Kinematic Analysis}

The solution of the forward kinematic problem of a parallel-kinematics robot is usually more complicated than that for its inverse, as the loop closure equations are highly nonlinear expressions of the actuation variables [27]. In this section we show, using an elimination technique, that the forward kinematics problem can be reduced to a polynomial equation governing the inplane kinematics and admitting six possible solutions. Since the forward kinematic problem does not admit a closed form solution, the inverse kinematic solution is used throughout this work for the purpose of design, analysis, and verification of the experimental data.

Consider the schematic of the XYZ MEMS shown in Fig. 2. For simplicity we consider the system to have three identical kinematic chains that are the result of consecutive rotations of $120^{\circ}$ with respect to each other. The chains are labeled as $\boldsymbol{A}, \boldsymbol{B}$ and $\boldsymbol{C}$. The actuated distance of each chain is $\lambda_{i}(i=1$ to 3$)$. The lengths of the cranks of the four-bar mechanisms and that of the outof-plane links are $L_{1}$ and $L_{2}$ respectively and the angular displacement of each four-bar mechanism is $\theta_{i}$. $\alpha$ is the angle formed by the out-of-plane links and the base (XY plane). The end-effector is an equilateral triangle with in-center radius $R$. Because of the symmetry of the chains, the base links of each chain can be thought to be tangential to a circle of radius $R_{b}$ (see Fig. 2).

To facilitate the kinematic analysis of this mechanism, we consider two modificatons: First, the end-effector is collapsed to a point $\boldsymbol{P}$. This is possible because the table undergoes no rotations. To accommodate this, the outer radius of the stage is reduced by $R$. As shown in Figure 3, the three fixed/base points of the kinematic chains, i.e., $\boldsymbol{A}_{\boldsymbol{o}}, \boldsymbol{B}_{\boldsymbol{o}}, \boldsymbol{C}_{\boldsymbol{o}}$, are now located on a circumscribing circle with radius $r=R_{b}-R$ and coordinates: 


$$
\begin{aligned}
& \left(X_{A 0}, Y_{A 0}, Z_{A 0}\right)=(0,0,0) \\
& \left(X_{B 0}, Y_{B 0}, Z_{B 0}\right)=\left(\frac{\sqrt{3}}{2} r, \frac{3}{2} r, 0\right) \\
& \left(X_{C 0}, Y_{C 0}, Z_{C 0}\right)=\left(-\frac{\sqrt{3}}{2} r, \frac{3}{2} r, 0\right)
\end{aligned}
$$

The directions of actuation are defined by the unit vectors:

$$
\hat{\boldsymbol{e}}_{1}=\hat{j}, \hat{\boldsymbol{e}}_{2}=-\frac{\sqrt{3}}{2} \hat{i}-\frac{1}{2} \hat{j}, \hat{\boldsymbol{e}}_{3}=+\frac{\sqrt{3}}{2} \hat{i}-\frac{1}{2} \hat{j}
$$

The second modification involves representing the parallelogram 4-bar mechanism with a single link. This is valid, provided that additional constraints are introduced to keep the direction of the projections of the out-of-plane links (now meeting at the point $\mathrm{P}$ ) in the XY plane invarient. Under these conditions, the link used to represent the 4-bar mechanism, can be viewed as a redundant link in the parallelogram 4-bar mechanism system, parallel to the crank and follower, connecting the base to the connector, and having the same length. Figure 3 shows the resulting simplification in the scheme of the mechanism.

$\boldsymbol{A}_{\boldsymbol{I}}, \boldsymbol{B}_{\boldsymbol{I}}, \boldsymbol{C}_{\boldsymbol{l}}$ are the contact points between the links representing the four-bar mechanism and the actuators. Their coordinates are a function of the actuator displacements, $\lambda_{\mathrm{i}}$, and the directions of actuators. They can be written as:

$$
\begin{aligned}
& \left(X_{A 1}, Y_{A 1}, Z_{A 1}\right)=\left(X_{A 0}, Y_{A 0}+\lambda_{1}, 0\right) \\
& \left(X_{B 1}, Y_{B 1}, Z_{B 1}\right)=\left(X_{B 0}-\frac{\sqrt{3}}{2} \lambda_{2}, Y_{B 0}-\frac{1}{2} \lambda_{2}, 0\right) \\
& \left(X_{C 1}, Y_{C 1}, Z_{C 1}\right)=\left(X_{C 0}+\frac{\sqrt{3}}{2} \lambda_{3}, Y_{C 0}-\frac{1}{2} \lambda_{3}, 0\right)
\end{aligned}
$$

The projected length $\delta$ of the out-of-plane links in the XY plane varies equally for all the chains, regardless the configuration of the machine. Therefore, points $\boldsymbol{A}_{2}, \boldsymbol{B}_{2}$ and $\boldsymbol{C}_{2}$, connecting the links representing the four-bar mechanism and the out-plane links, lie on the circumference of a circle in the XY plane, centered at the projection of $\boldsymbol{P}$ in the XY plane, and with a radius that equals the projected length $\delta$ of the out-of-plane links (which in turn, depends on the table elevation). Thus, the coordinates of points $\boldsymbol{A}_{2}, \boldsymbol{B}_{2}, \boldsymbol{C}_{2}$ are:

$$
\begin{aligned}
& \left(X_{A 2}, Y_{A 2}, Z_{A 2}\right)=\left(P_{x}, P_{y}-\delta, 0\right) \\
& \left(X_{B 2}, Y_{B 2}, Z_{B 2}\right)=\left(P_{x}+\frac{\sqrt{3}}{2} \delta, P_{y}+\frac{\delta}{2}, 0\right) \\
& \left(X_{C 2}, Y_{C 2}, Z_{C 2}\right)=\left(P_{x}-\frac{\sqrt{3}}{2} \delta, P_{y}+\frac{\delta}{2}, 0\right)
\end{aligned}
$$

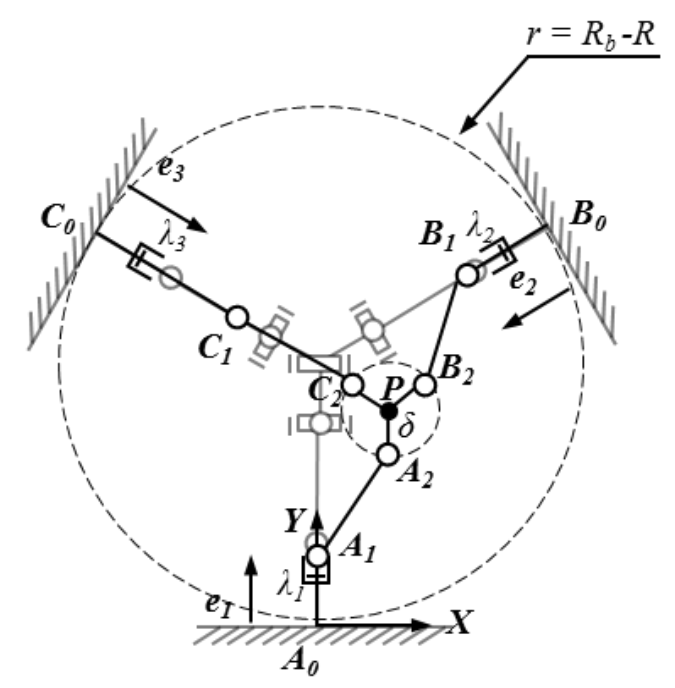

Fig. 3. Final kinematics transformation in which the end effector is collapsed to a point with the constraint that its orientation remains invariant. 
The algebraic system of equations describing the kinematics problem is obtained by constrainting the distances of segments $\overline{\boldsymbol{A}_{\mathbf{1}} \boldsymbol{A}_{\mathbf{2}}}, \overline{\boldsymbol{B}_{\mathbf{1}} \boldsymbol{B}_{\mathbf{2}}}, \overline{\boldsymbol{C}_{\mathbf{1}} \boldsymbol{C}_{\mathbf{2}}}$ to be equal to $L_{1}$. We get:

$$
\begin{gathered}
\left(P_{x}-A_{0 x}\right)^{2}+\left(P_{y}-A_{0 y}-\left(\lambda_{1}+\delta\right)\right)^{2}-L_{1}^{2}=0 \\
\left(P_{x}-B_{0 x}+\frac{\sqrt{3}}{2}\left(\lambda_{2}+\delta\right)\right)^{2}+\left(P_{y}-B_{0 y}+\frac{1}{2}\left(\lambda_{2}+\delta\right)\right)^{2}-L_{1}^{2}=0 \\
\left(P_{x}-C_{0 x}-\frac{\sqrt{3}}{2}\left(\lambda_{3}+\delta\right)\right)^{2}+\left(P_{y}-C_{0 y}+\frac{1}{2}\left(\lambda_{3}+\delta\right)\right)^{2}-L_{1}^{2}=0
\end{gathered}
$$

With unknowns $P_{x}, P_{y}$ and $\delta$. Further elimination of the variables $P_{\mathrm{x}}$ and $P_{y}$ yields a sixth order polynomial equation in the parameter $\delta$. The coefficients of this polynomial are functions of the coordinates of the fixed points $\boldsymbol{A}_{o}, \boldsymbol{B}_{o}, \boldsymbol{C}_{\boldsymbol{o}}$ and the displacements $\lambda_{i}$ of the actuators. The particular equation governing the direct kinematics of the stage (see Appendix A) can be obtained by substitution of the base points coordinates into Eq. (5) with the trial values for the mechanism dimensions of $r=2.5$ $\mathrm{mm}, L_{1}=1, L_{2}=0.7071 \mathrm{~mm}$ and using software for symbolic computing ${ }^{1}$. Although there are six solutions to the forward kinematics problem, we are interested in those that are physically realizable, and thus corresponding to the values of $P_{\mathrm{x}}$ and $P_{y}$ being real and the projected distance $\delta$ being positive and less than $L_{2}$. From here, the height $P_{z}$ of point $\boldsymbol{P}$ above the XY plane is calculated as the positive root of:

$$
P_{z}=\sqrt{L_{2}^{2}-\delta^{2}}
$$

This completes the forward position kinematics.

\section{B. Inverse and Velocty Kinematic Analysis}

The inverse kinematics problem of the XYZ parallel kinematics is stated as: Given the coordinates $\left(P_{x}, P_{y}, P_{z}\right)$ of point $\mathbf{P}$ on the table with respect to the fixed coordinate system, find the values of $\lambda_{1}, \lambda_{2}, \lambda_{3}$. From the value of $P_{z}$ we know that $\delta$, the projection of the length $L_{2}$ on the $\mathrm{XY}$ plane is the positive root of:

$$
\delta=\sqrt{L_{2}^{2}-P_{z}^{2}}
$$

The solution of the inverse kinematics problem is arrived at by substitution of Eq. (7) into the system of equations (5) and solving for $\lambda_{1}, \lambda_{2}, \lambda_{3}$ independently in each of the equations. Therefore, we have:

$$
\begin{aligned}
\lambda_{1}= & P_{y}-\sqrt{l_{2}^{2}-P_{z}^{2}} \pm \sqrt{l_{1}^{2}-\left(P_{x}-X_{\mathrm{A} 0}\right)^{2}}-Y_{\mathrm{A} 0}, \\
\lambda_{2}= & \frac{1}{2}\left(-\sqrt{3} P_{x}-P_{y}-2 \sqrt{l_{2}^{2}-P_{z}^{2}}+\sqrt{3} X_{\mathrm{B} 0}+Y_{\mathrm{B} 0}\right) \pm \frac{1}{2}\left\{\left[4 l_{1}^{2}-P_{x}^{2}-3 P_{y}^{2}-2 \sqrt{3} P_{y} X_{\mathrm{B} 0}-X_{\mathrm{B} 0}^{2}+6 P_{y} Y_{\mathrm{B} 0}+\right.\right. \\
& \left.\left.2 \sqrt{3} X_{\mathrm{B} 0} Y_{\mathrm{B} 0}-3 Y_{\mathrm{B} 0}^{2}+2 P_{x}\left(\sqrt{3} P_{y}+X_{\mathrm{B} 0}-\sqrt{3} Y_{\mathrm{B} 0}\right)\right]\right\}^{1 / 2} \\
\lambda_{3}= & \frac{1}{2}\left(\sqrt{3} P_{x}-P_{y}-2 \sqrt{l_{2}^{2}-P_{z}^{2}}-\sqrt{3} X_{\mathrm{C} 0}+Y_{\mathrm{C} 0}\right) \pm \frac{1}{2}\left\{\left[4 l_{1}^{2}-P_{x}^{2}-3 P_{y}^{2}+2 \sqrt{3} P_{y} X_{\mathrm{C} 0}-X_{\mathrm{C} 0}^{2}+6 P_{y} Y_{\mathrm{C} 0}-\right.\right. \\
& \left.\left.2 \sqrt{3} X_{\mathrm{C} 0} Y_{\mathrm{C} 0}-3 Y_{\mathrm{C} 0}^{2}+2 P_{x}\left(-\sqrt{3} P_{y}+X_{\mathrm{C} 0}+\sqrt{3} Y_{\mathrm{C} 0}\right)\right]\right\}^{1 / 2}
\end{aligned}
$$

Each of the $\lambda_{i}$ can take two values, corresponding to the intersections of a circle of radius $L_{l}$ with the directions of actuation. Knowledge of the range of permissible values for $\lambda_{i}$ permits the identification of the correct solution. This completes the inverse position kinematics. Next, we address the velocity kinematics problem by computing the Jacobian matrices relating the velocities of all the members of the manipulator to the velocity of the actuators by differentiating Eq. (5). Here, the position of end-effector is known. The instantaneous kinematics problem is relevant to our subsequent discussion on the workspace and dynamics of the manipulator.

Because of the relatively small angular displacement of the joints, the kinematics can be linearized around the nominal configuration of the machine resulting in an invariant Jacobian matrix of the system $J_{l}$ relating the linear displacement vector of the end-effector from its nominal position to the displacements of the actuators by the following relationship.

$$
\left[\begin{array}{l}
\Delta \lambda_{1} \\
\Delta \lambda_{2} \\
\Delta \lambda_{3}
\end{array}\right]=\left[\begin{array}{lll}
\frac{\partial \lambda_{1}}{\partial x} & \frac{\partial \lambda_{1}}{\partial y} & \frac{\partial \lambda_{1}}{\partial z} \\
\frac{\partial \lambda_{2}}{\partial x} & \frac{\partial \lambda_{2}}{\partial y} & \frac{\partial \lambda_{2}}{\partial z} \\
\frac{\partial \lambda_{3}}{\partial x} & \frac{\partial \lambda_{3}}{\partial y} & \frac{\partial \lambda_{3}}{\partial z}
\end{array}\right]\left[\begin{array}{l}
\Delta x \\
\Delta y \\
\Delta z
\end{array}\right]=J_{1}^{-1}\left[\begin{array}{c}
\Delta x \\
\Delta y \\
\Delta z
\end{array}\right]
$$

\footnotetext{
${ }^{1}$ Mathematica ${ }^{\circledR}$ by Wolfram Research Inc.
} 
Proceeding in a similar manner, for the hinges of the 4-bar mechanisms of each chain, we have

$$
\left[\begin{array}{c}
\Delta \theta_{1} \\
\Delta \theta_{2} \\
\Delta \theta_{3}
\end{array}\right]=\left[\begin{array}{lll}
\frac{\partial \theta_{1}}{\partial x} & \frac{\partial \theta_{1}}{\partial y} & \frac{\partial \theta_{1}}{\partial z} \\
\frac{\partial \theta_{2}}{\partial x} & \frac{\partial \theta_{2}}{\partial y} & \frac{\partial \theta_{2}}{\partial z} \\
\frac{\partial \theta_{3}}{\partial x} & \frac{\partial \theta_{3}}{\partial y} & \frac{\partial \theta_{3}}{\partial z}
\end{array}\right]\left[\begin{array}{l}
\Delta x \\
\Delta y \\
\Delta z
\end{array}\right]=J_{2}^{-1}\left[\begin{array}{c}
\Delta x \\
\Delta y \\
\Delta z
\end{array}\right]
$$

Similarly, for the out-of-plane hinges, we have,

$$
[\Delta \alpha]=\left[\begin{array}{lll}
\frac{\partial \alpha_{1}}{\partial x} & \frac{\partial \alpha_{1}}{\partial y} & \frac{\partial \alpha_{1}}{\partial z}
\end{array}\right]\left[\begin{array}{l}
\Delta x \\
\Delta y \\
\Delta z
\end{array}\right]=J_{3}^{-1}\left[\begin{array}{c}
\Delta x \\
\Delta y \\
\Delta z
\end{array}\right]
$$

For the symmetric design of the mechanism in this paper, we have the following Jacobian matrices at the nominal configuration expressed as functions of the design parameters $L_{1}$ and $L_{2}$ and the initial elevation angle $\alpha_{0}$ :

$$
\begin{aligned}
& J_{1}^{-1}=\left[\begin{array}{ccc}
0 & 1 & \tan \alpha_{0} \\
-\frac{\sqrt{3}}{2} & -\frac{1}{2} & \tan \alpha_{0} \\
\frac{\sqrt{3}}{2} & -\frac{1}{2} & \tan \alpha_{0}
\end{array}\right], J_{2}^{-1}=\left[\begin{array}{ccc}
-\frac{1}{L_{1}} & 0 & 0 \\
\frac{1}{2 L_{1}} & \frac{-\sqrt{3}}{2 L_{1}} & 0 \\
\frac{1}{2 L_{1}} & \frac{\sqrt{3}}{2 L_{1}} & 0
\end{array}\right] \\
& J_{3}^{-1}=\left[\begin{array}{lll}
0 & 0 & \frac{\sec \alpha_{0}}{L_{2}}
\end{array}\right]
\end{aligned}
$$

The linearized form of the instantaneous kinematics has the following properties:

- Near to the nominal configuration, the Z (out-of-plane) motion of the manipulator is dependent on the elevation angle $\alpha_{0}$, but independent of the lengths $L_{1}$ and $L_{2}$.

- The summation of the angular displacements of all 4-bar mechanisms is zero.

- Any displacements of the end-effector in an XY plane (with constant $Z$ height) in the manipulator's workspace is defined by $\Delta \lambda 1+\Delta \lambda_{2}+\Delta \lambda_{3}=C$, where $C$ is constant.

Motion of the end-effector along the $\mathrm{Z}$ axis from the nominal position corresponds to displacement of the actuators such that $\Delta \lambda_{1}=\Delta \lambda_{2}=\Delta \lambda_{3}$.

\section{Static and Dynamic Analysis}

Lagrange's equation, derived from the Hamiltonian principle, is used to calculate the stiffness matrix, vibration modes and natural frequencies of the manipulator. We have,

$$
\frac{d}{d t}\left(\frac{\partial T}{\partial \dot{x}_{i}}\right)-\frac{\partial T}{\partial x_{i}}+\frac{\partial V}{\partial x_{i}}=Q_{i}, i=1,2,3
$$

Where $T$ is the total kinetic energy in the system, $V$ is the potential energy of the system including the strain energy stored in the elastic elements of the system and the potential of any conservative force, $x_{i}$ is the linearly independent set of generalized coordinates $\left(x, y\right.$ and $z$ ) and $Q_{i}$ represents the generalized non conservative forces (the external forces at the end-effector of the mechanism). For the purpose of analysis, the manipulator is assumed to have all of its mass concentrated at the moving table and all of flexible elements to be perfectly elastic. Any non-conservative forces in the system are neglected. From the linearized kinematics of the stage, we can relate the displacements of the generalized coordinates $\Delta \mathbf{x}=[\Delta x, \Delta y, \Delta z]^{\mathbf{T}}$ to the displacements of the actuators $\Delta \mathbf{q}=\left[\Delta \lambda_{1}, \Delta \lambda_{2}, \Delta \lambda_{3}\right]^{\mathbf{T}}$ from their nominal positions as $\Delta \mathbf{q}=J_{1}^{-1} \Delta \mathbf{x}$. Since the mass of the system is assumed to be concentrated at the table, which undergoes pure translation, the total kinetic energy of the system is approximated as:

$$
T=\frac{1}{2} m\left[\begin{array}{lll}
\Delta \dot{x} & \Delta \dot{y} & \Delta \dot{z}
\end{array}\right]\left[\begin{array}{c}
\Delta \dot{x} \\
\Delta \dot{y} \\
\Delta \dot{z}
\end{array}\right]
$$

The potential energy of the system is given by the strain energy stored as elastic deformations of the flexible elements of the device, i.e., the folded leaf-spring, the in-plane and the out-of-plane hinges. The strain energy stored in a leaf of the folded spring suspension of the $\mathrm{i}^{\text {th }}$-kinematic chain is:

$$
V=\frac{1}{2} K_{1} \Delta \lambda_{i}^{2}, i=1,2,3
$$


Where $K_{1}$ is the linear stiffness of an individual folded leaf-spring. Since the folded leaf-springs we use in our designs have 4 leaves that simultaneously deformed, $V=2 K_{1} \Delta \lambda_{i}{ }^{2}$. The energy stored in all the folded leaf-spring suspensions (there is one for each kinematic chain) can be related to the displacement of the end effector through the Jacobian matrix as:

$$
\begin{aligned}
& V_{1}=2 K_{1}\left[\begin{array}{lll}
\Delta \lambda_{1} & \Delta \lambda_{2} & \Delta \lambda_{3}
\end{array}\right] \cdot\left[\begin{array}{l}
\Delta \lambda_{1} \\
\Delta \lambda_{2} \\
\Delta \lambda_{3}
\end{array}\right] \\
& =2 K_{1}\left[\begin{array}{lll}
\Delta x & \Delta y & \Delta z
\end{array}\right] \cdot\left[J_{1}^{-\mathrm{T}} J_{1}^{-1}\right] \cdot\left[\begin{array}{c}
\Delta x \\
\Delta y \\
\Delta z
\end{array}\right]
\end{aligned}
$$

Each chain has also a 4-bar mechanism with 4 flexural hinges. Further, we expect two sets of 2 small-flexural pivots at the two ends of the out-of-plane link. Hence, the same expression applies to the energy stored in these elements. Thus, the total potential energy of the system becomes the addition potential energy store at each flexural element of the manipulator:

$$
V=\sum_{i=1}^{3} V_{i}=\left[\begin{array}{lll}
\Delta x & \Delta y & \Delta z
\end{array}\right] \cdot 2\left[\sum_{i=1}^{3} K_{i} J_{i}^{-\mathrm{T}} J_{i}^{-1}\right] \cdot\left[\begin{array}{c}
\Delta x \\
\Delta y \\
\Delta z
\end{array}\right]
$$

Where $K_{1}, K_{2}$ and $K_{3}$ are the stiffnesses of the folded leaf-spring, the in-plane hinges in 4-bar system and the out-of-plane hinges at the ends of the out-of-plan link, respectively. Taking Eq. (14) and Eq. (17) and substituting into the Lagrange's equation, we get the dynamics equation of the stage as:

$$
m\left[\begin{array}{c}
\Delta \ddot{x} \\
\Delta \ddot{y} \\
\Delta \ddot{z}
\end{array}\right]+K\left[\begin{array}{c}
\Delta x \\
\Delta y \\
\Delta z
\end{array}\right]=\left[\begin{array}{c}
F_{x} \\
F_{y} \\
F_{z}
\end{array}\right], K=4\left[\sum_{i=1}^{3} K_{i} J_{i}^{-\mathrm{T}} J_{i}^{-1}\right]
$$

Where $\left[\begin{array}{lll}F_{x} & F_{y} & F_{z}\end{array}\right]^{\mathrm{T}}$ is the vector of external forces at the moving table. Eq. (18) represents the dynamics of a 3DOF harmonic oscillator. An expression for the stiffness of the system as a function of the design parameters $L_{l}, L_{2}$, and $\alpha_{0}$, is obtained by substitution of the Jacobian matrices of Eq. (12) into Eq. (18). Namely:

$$
K=\left[\begin{array}{ccc}
6\left(K_{1}+K_{2} / L_{1}^{2}\right) & 0 & 0 \\
0 & 6\left(K_{1}+K_{2} / L_{1}^{2}\right) & 0 \\
0 & 0 & 4 K_{3} \sec \left[\alpha_{0}\right]^{2} / L_{2}{ }^{2+} \\
0 & & 12 K_{1} \tan \left[\alpha_{0}\right]^{2}
\end{array}\right]
$$

The linearized form of the stiffness of the system has the following properties:

- Near the nominal configuration, the in-plane stiffness is proportional to the stiffnesses of the folded leaf-spring suspensions, $K_{1}$, and the 4-bar hinges, $K_{2}$. It is inversely proportional to the square of the length of the 4-bar mechanism crank $L_{1}$.

- The out-of-plane stiffness is proportional to the stiffness of the folded leaf-spring suspensions, $K_{l}$, and the out-of-plane hinges, $K_{3}$. It is inversely proportional to the square of the length of the out-of-plane links $L_{2}$.

- High values of the initial elevation angle $\alpha_{0}$ result in high mechanical advantage along the $\mathrm{Z}$ direction which causes the out-of-plane stiffness of the manipulator to be high.

To select the design parameters that ensure meeting the mechanical advantage or motion requirements (given the duality between them), it is desirable to match the force-stroke capability of the comb actuators with the stiffness of the manipulator structure. To exemplify this, the force-displacement relationship for the out-of-plane motion of the manipulator can be written from Eq. (18) as:

$$
\frac{F_{z}}{\Delta z}=4 K_{3} \sec [\alpha]^{2} / L_{2}^{2}+12 K_{1} \tan [\alpha]^{2}
$$

From the linearized kinematics of the manipulator, we have:

$$
\frac{\Delta \lambda}{\Delta z}=\tan \left(\alpha_{0}\right)
$$

Where $\Delta \lambda$ are the displacement applied by all the actuators to move the stage an amount $\Delta z$. Also, from an energetic point of view, the energy required to push the three actuators must be equal to the energy invested in displacing the moving table in the $\mathrm{z}$ direction and thus, we get:

$$
3 F_{\lambda} \cdot \Delta \lambda=F_{z} \cdot \Delta z
$$

Where $F_{\lambda}$ is the force applied by each actuator to lift the moving stage an amount $\Delta z$. It should be notice that Eq. (22) is consistent with the duality between displacement and force in the manipulator, namely: 


$$
\mathbf{F}_{\mathbf{x}}=\left(J_{1}^{-1}\right)^{T} \mathbf{F}_{\lambda}
$$

Vectors $\mathbf{F}_{\mathrm{x}}$ and $\mathbf{F}_{\lambda}$ in (23) correspond to the forces produced by the actuators and the forces transmitted to the end-effector, respectively. Substituting Eqs. (21) and (22) into Eq. (20), the out-of-plain motion of the manipulator can be expressed in terms of the force-displacement characteristics of the actuators as:

$$
\frac{F_{\lambda}}{\Delta \lambda}=\frac{1}{3} \cdot\left[4 K_{3} \csc [\alpha]^{2} / L_{2}^{2}+12 K_{1}\right]
$$

Figure 4 shows the parametric design space $L_{2}-\alpha_{0}$ in which Eq. (24) represents a design constraint resulting from matching the force-displacement characteristics of different comb-drives to the stiffness of the manipulator. Multiple design constraints are shown corresponding to different values of actuation forces. Each of these constraints divides the space into two regions. The region above to the constraints represents selections of lengths $L_{2}$ and angles $\alpha_{0}$ in which the actuators have enough force to overcome the out-of-plane stiffness of the structure, reach their maximum stroke and balance an opposing vertical force at the end effector. Points across this region should be selected to meet different spatial and mechanical advantage requirements. Conversely, the region below corresponds to points where the stiffness of the structure is high enough so that the actuators cannot reach the maximum stroke. Finally, points lying on the constraint represent the limit in which all the force generated by the actuators is used to overcome the stiffness of the structure and no force is available to the manipulator for external work.

\section{Design for Physical Realization}

In this section, we assess the feasibility and expected performance of a PK-XYZ-MEMS stage. In our previous work [3], [28] we have designed several elements that can be used in realizing the proposed scheme and optimized processes (taking into consideration capabilities and limits of our facilities) for their fabrication. These include in-plane hinges, leaf-springs, out-ofplane hinges, comb drives, sensing combs, and parallelogram 4-bar mechanism modules. The idea is to use these elements and a typically available SOI substrate to layout the design of a general-purpose stage with a workspace volume that can be embed a cube with side $20 \mu \mathrm{m}$ and have a natural frequency of about $500-1000 \mathrm{~Hz}$ so that a sufficiently large frequency band is available for accommodating different positioning and probing applications. To do so, we must decide the parameters $L_{1}, L_{2}$, and $\alpha_{0}$, of the mechanism so as to match the stroke and force capabilities of the comb drives, the elastic range of the folded leaf-spring suspension, the 4-bar hinges, the out-of-plain joints, and the dimensional limits of available SOI structures. This exercise, therefore, not only serves to demonstrate the feasibility of designing stages with reasonable and useful characteristics, but also elucidates a design methodology for PK-XYZ-MEMS stages.

\section{A. Folded Spring Suspension Design}

The suspension structure is designed to have large compliance in the direction of actuation and high stiffness in the parasitic direction to prevent side instabilities. Figure 5 shows a solid model of a kinematic chain in the mechanism, extracted from a detailed solid model of the designed system. In it are shown the different flexible elements of the chain: the folded leaf springs, the out-of-plane springs, and the in-plane four-bar mechanism flexural hinges. The folded leaf-spring suspension used to support

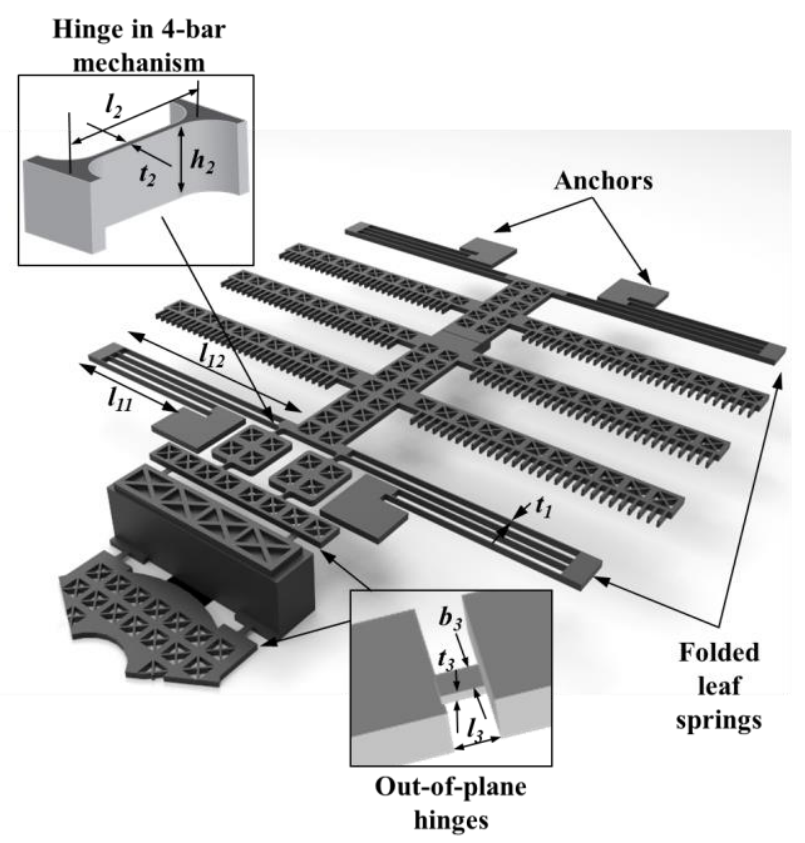

Fig. 5. Solid model of one kinematic chain. Parameters of the different flexible elements in the chain are identified and explained with inserts. 
the device can be analyzed by elementary beam deflection theory. The stiffness of a clamped-clamped beam along the motion direction $K_{d}$ and lateral direction $K_{l}$ can be expressed as:

$$
K_{d}=\frac{2 E h_{1} t_{1}^{3}}{l^{3}}, K_{l}=\frac{2 E h_{1} t_{1}}{l}
$$

where $l$ is the length of the leaf, $t_{l}$ is its thickness and $h_{l}$ is its height. For one folded leaf-spring, the overall stiffness in the direction of motion and lateral direction is computed by combining the stiffness of two clamped beams in series. The stiffness of the two beams (with lengths $l_{11}$ and $l_{12}$ ) are $K_{d 1}=4 E h_{1} t_{1}{ }^{3} / l_{11}{ }^{3}, K_{d 2}=4 E h_{1} t_{1}{ }^{3} / l_{12}{ }^{3}$ and thus, the overall stiffness of the folded leaf-spring is.

$$
K_{1}=\frac{K_{d 1} K_{d 2}}{K_{d 1}+K_{d 2}}=\frac{4 E h_{1} t_{1}^{3}}{l_{11}^{3}+l_{12}{ }^{3}},
$$

Similarly, the overall stiffness in the lateral direction is

$$
K_{1 l}=\frac{K_{l 1} K_{l 2}}{K_{l 1}+K_{l 2}}=\frac{4 E h_{1} t_{1}}{l_{11}+l_{12}}
$$

The chosen dimensions for our folded leaf-spring system are $l_{11}=1.375 \mathrm{~mm}, l_{12}=1.895 \mathrm{~mm}, t_{1}=8 \mu \mathrm{m}, h_{1}=90 \mu \mathrm{m}$ and the stiffness in the direction of actuation becomes $K_{l}=0.002938 \mathrm{~N} / \mathrm{mm}$ and the stiffness ratio $K_{l l} / K_{l}$ is equal to $\left(l_{11}{ }^{3}+l_{12}{ }^{3}\right) /\left(l_{11}+\right.$ $\left.l_{12}\right) t_{1}{ }^{2}=44900$.

\section{B. 4-Bar Mechanism Hinges}

The insert in Figure 5 shows a solid model of the in-plane four-bar mechanism flexural hinge with relevant parameters. An approximation of the resistance of an in-plane flexure of the four-bar mechanism to horizontal bending [29] is obtained as:

$$
K_{2}=\frac{E I_{2}}{l_{2}}=\frac{E h_{2} t_{2}{ }^{3}}{12 l_{2}}
$$

Where $E$ is the young modulus, $h_{2}$ is the width, $t_{2}$ is the thickness and $l_{2}$ is the length of the flexural pivot. The maximum bending torque applied to the hinge is

$$
M_{\max }=\frac{2 \sigma_{p} I_{2}}{t_{2}}
$$

Where $I_{2}=h_{2} t_{2}^{3} / 12$ is the moment of inertia of the flexure hinge about the axis of rotation and $\sigma_{p}$ is the fracture strength of single crystal silicon. The rotational range limit for the in-plane flexural hinges is given by

$$
\theta_{\max }=M_{\max } C_{z}
$$

For the flexure hinges used in our design, we have the following parameters: $l_{2}=100 \mu \mathrm{m}, t_{2}=6 \mu \mathrm{m}$ and $h_{2}=90 \mu \mathrm{m}$. The young modulus of single crystal silicon is $150 \mathrm{GPa}$ and its elastic limit is about $7 \mathrm{GPa}$. With these dimensions and material properties, the stiffness and maximum rotation range of the hinges are $2.43 \times 10^{-3} \mathrm{~N} \mathrm{~mm} / \mathrm{rad}$ and $1.5 \mathrm{rad}$ respectively. This rotation translates into a maximum displacement range of $490 \mu \mathrm{m}$ of the 4 bar structure.

\section{Out-of-Plane hinges}

In our design, the out-of-plain links (shown in Figure 5) are connected by a pair of small flexural pivots to both the 4-bar structure and the moving table. The torsional pivot resistance to bending is given by Eq. (28) as

$$
K_{3}=\frac{E I}{l_{3}}=\frac{E b_{3} t_{3}^{3}}{12 l_{3}}
$$

Where $E$ is the young modulus, $b_{3}$ is the width, $t_{3}$ is the thickness and $l_{3}$ is the length of the flexural pivot. The maximum bending moment that can be applied to each flexural pivot is given by Eq. (29). Thus, the rotational range of the out-of plane hinges is given by

$$
\alpha_{\max }=\frac{M_{\max }}{K_{3}}=\frac{2 \sigma_{p} l_{3}}{t_{3} E}
$$

The parameters of our design become $l_{3}=100 \mu \mathrm{m}, t_{3}=10 \mu \mathrm{m}, b_{3}=50 \mu \mathrm{m}$, and the stiffness and maximum rotation range of the hinges are $6.25 \times 10^{-3} \mathrm{~N} \mathrm{~mm} / \mathrm{rad}$ and $0.94 \mathrm{rad}$ respectively.

\section{Comb-Drive Actuators}

The comb drive actuators must provide sufficient force to overcome the stiffness of the folded leaf-spring suspension, the 4bar mechanism and the out-of-plane hinges. The force provided by a comb drive is given by the formula

$$
F=n \frac{\varepsilon_{0} h V^{2}}{g}
$$

Where $n$ is the number of fingers, $h$ is the height of a finger and $g$ is the gap distance between to neighboring fingers. Our comb design has 360 fingers with a $5 \mu \mathrm{m}$ gaps and $100 \mu \mathrm{m}$ heights, generating a force of $1 \mathrm{mN}$ at $130 \mathrm{~V}$. MEMS probes, 
previously designed by our laboratory used similar electrostatic comb actuators and folded leaf spring designs and were found to have constant stiffness through measured displacements of $40 \mu \mathrm{m}$ within $5 \mathrm{~nm}$ resolution [3]. Besides electrostatic forces along the direction of motion, there are also electrostatic forces pulling the fingers together. According to [30], if the lateral stiffness of the folded springs $K_{l l}$ is large compared with the axial stiffness $K_{l}\left(K_{l l} \gg K_{l}\right)$, the maximum displacement $\Delta \lambda$ that can be obtained before pull-in will occur is

$$
\Delta \lambda=g \sqrt{\frac{k_{1 l}}{2 k_{1}}}-\lambda_{0}
$$

Where $\lambda_{0}$ is the initial overlap between the fingers of the stator and the rotor. Based to this, and ignoring other effects, the design is capable of undergoing displacements of about $700 \mu \mathrm{m}$ (far outside our designed range of motion) before experiencing pull-in instabilities.

\section{E. Selection of Kinematic Parameters and Dimensions for Stage.}

One device layer (DL1) has to accommodate the actuation, the leaf-springs and the 4-bar linkage, it will therefore carry suspended structures with large lateral dimensions. Further, the leaf-springs in this layer must resist the twisting forces that result from converting in-plain actuation to out-of-plane motion. For these reasons, it was decided to make this layer thicker that the other device layer (DL2) which only carries the end-effector. Initially, we selected a 50 micron device layer for DL1 and a 25 micron layer for DL2. After a number of design iterations, in which various design parameters were assessed, it was apparent that a device layer thickness greater than 70 microns was needed for DL1 to resist unwanted torsional deflection of the leafsprings and deflection of the entire structure under its own weight. Based on this requirement, an "oreo" wafer, Ultrasil D4-6110, with device layer thicknesses of $90 \mu \mathrm{m}$ and $27 \mu \mathrm{m}$, handle layer thickness of $300 \mu \mathrm{m}$, and BOX layers' thickness of $0.5 \mu \mathrm{m}$ was selected as the target substrate for this design.

To accommodate the lateral dimensions of the leaf-springs and the comb-actuators, and to provide space for traces routing power and signals to and from the actuating and sensing combs, the parameter ' $r$ ' (see Figures 2 and 3) was chosen to be $2 \mathrm{~mm}$, with $R_{b}=2.5 \mathrm{~mm}$ and $R=0.5 \mathrm{~mm}$. Next, the parameters $L_{1}$ (length of the 4-bar crank), $L_{2}$ (length of the out-of-plane link, and the elevation angle, $\alpha_{0}$ were determined by trying to simultaneously meet objectives of stiffness/structural resonance frequency of the stage $(500-1000 \mathrm{~Hz})$, workspace objectives, while being constrained by the force and displacement output of the actuators, the dimensions chosen for the substrate and the overall stage. Based on these considerations, $L_{1}=0.5 \mathrm{~mm}, L_{2}=0.589 \mathrm{~mm}$ and $\alpha_{0}$ $=0.6$ radians, were chosen as the parameters for the mechanism design.

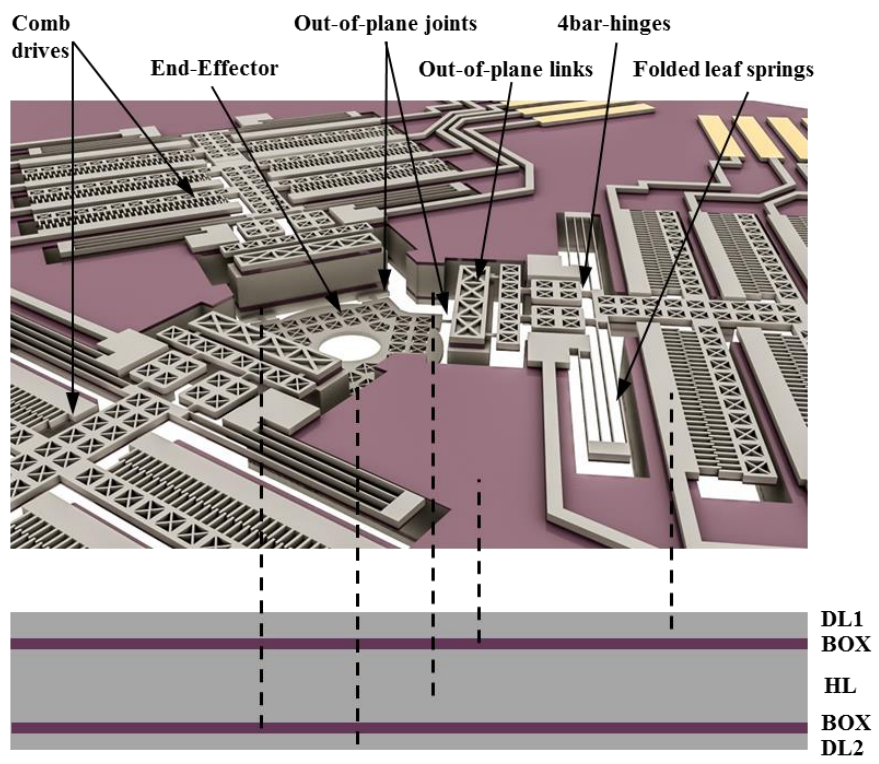

Fig. 6.(a) CAD rendered image of the PK-XYZ-MEMS showing the mapping of the elements of the device to the layers of the substrate. 
Based on these component designs and values for the stage parameters, a detailed design was laid in the three layers of the double SOI wafer. Figure (6) shows a solid model view of the design of the stage when the kinematic chains of Figure (5) are assembled and mapped into the different layers of the substrate. To reduce the mass of the stage while maintaining stiffness, the moving parts are patterned with a truss-like structure, resulting on a total mass of $3.655 \times 10^{-3} \mathrm{mg}$.

\section{E. Assessment of Workspace of PK-XYZ-MEMS}

With the above parameters and dimensions, the workspace for the stage would traditionally be computed by solving the forward position kinematic equations for end-effector locations while iterating through all feasible combinations of displacements of the actuated joints. Other constraints, such as the feasible displacements of the unactuated joints, may also be checked while determining the boundaries of the workspace. Here, because we have a parallel kinematic mechanism, and because the range of motion of the actuators is so much smaller than the dimensions of the links of the machine, we can directly use the Jacobian of the stage, computed when all actuators are the center of their motion range, as an invariant relationship between the joint displacements and end-effector displacements.

$$
\left[\begin{array}{c}
\Delta x \\
\Delta y \\
\Delta z
\end{array}\right]=J_{1}^{-1}\left[\begin{array}{l}
\Delta \lambda_{1} \\
\Delta \lambda_{2} \\
\Delta \lambda_{3}
\end{array}\right]
$$

Considering the range of motion of the actuated joints to be $25 \mu \mathrm{m}$, the position of the end-effector at the center of its workspace is found by solving the position forward kinematics, given in Equation (5). This is used to find the elevation angle, $\alpha_{0}$, of the out-of-plane link, and using Equation (12) we get:
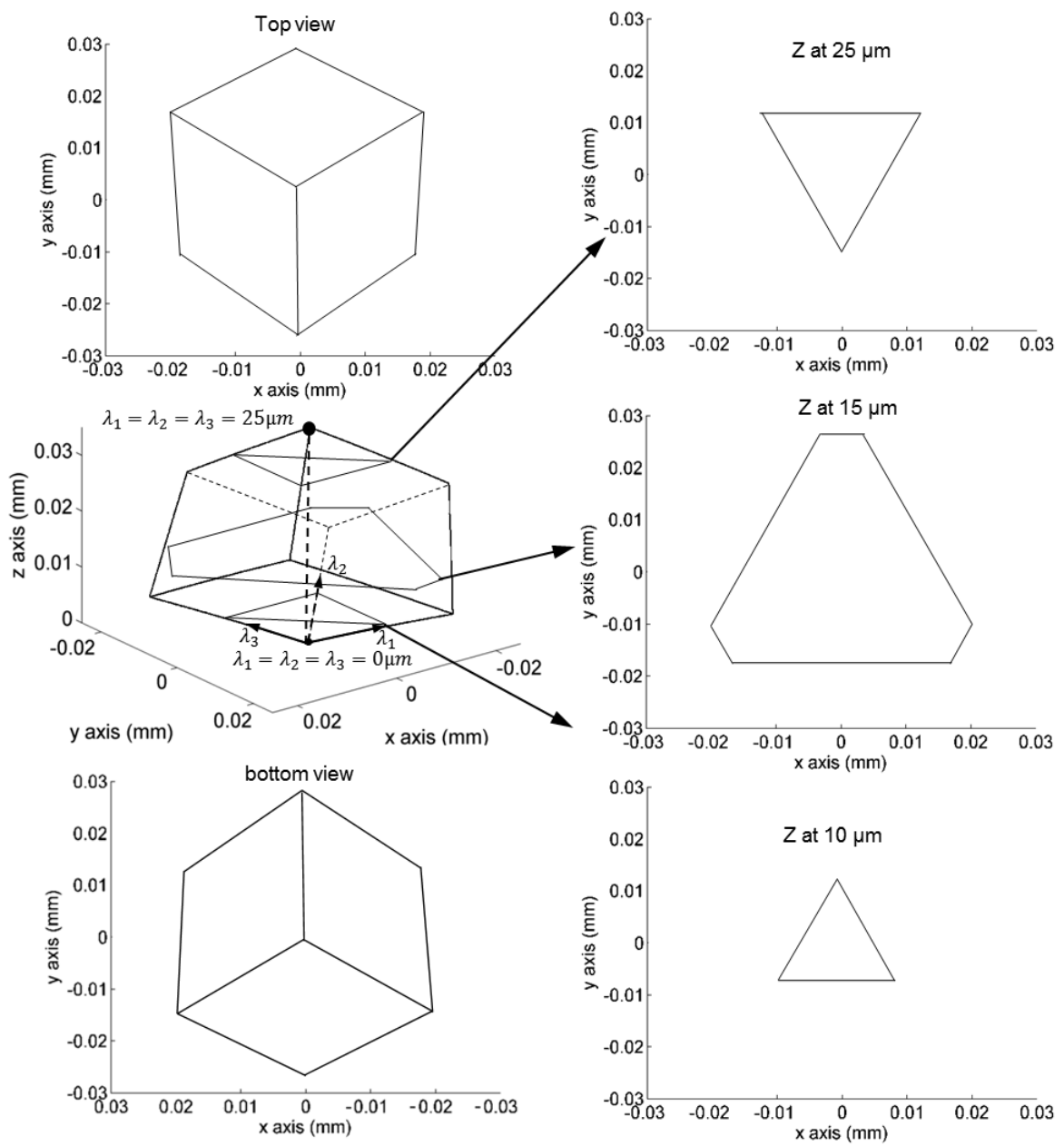

Fig. 7. Work space of the end effector of the $X Y Z$ stage and cross region on $X Y$ plane at different $Z$ height. 


$$
J_{1}^{-1}=\left[\begin{array}{ccc}
0 & 1 & 0.677 \\
-\frac{\sqrt{3}}{2} & -\frac{1}{2} & 0.677 \\
\frac{\sqrt{3}}{2} & -\frac{1}{2} & 0.677
\end{array}\right]
$$

Fig, (6) shows the workspace for the stage. It can be seen that the volume of the workspace for a theoretical displacement of the actuators of $25 \mu \mathrm{m}$ is $2432.5 \mu \mathrm{m}^{3}$ and the highest $\mathrm{Z}$ displacement is at $36 \mu \mathrm{m}$. Further, the workspace has the shape of a cube standing on one of its vertices. The constraints generating the boundaries of the workspace are also indicated in the figure.

The parasitic angular errors of the stage are calculated using finite element simulations. To obtain an estimate of the maximum value, the errors are computed for the end-effector moving along edges of the workspace on a path starting from the nominal point, passing through a point of maximum lateral displacement and ending at the point of highest z-displacement. A maximum tilting error of 2.61 milliradians was estimated from these simulations (see Appendix B).

\section{F. Dynamic behavior}

To obtain the theoretical modal frequencies of the system, the forces at the end-effector are assumed to be zero and the dynamic equation of the system becomes:

$$
m\left[\begin{array}{c}
\Delta \ddot{x} \\
\Delta \ddot{y} \\
\Delta \ddot{z}
\end{array}\right]+K\left[\begin{array}{c}
\Delta x \\
\Delta y \\
\Delta z
\end{array}\right]=\left[\begin{array}{l}
0 \\
0 \\
0
\end{array}\right]
$$

This corresponds to the dynamics of a 3DOF harmonic oscillator. The eigenvalues of $K$ will give us the mode-shapes of the system and the model frequency can be written as a function of the eigenvalues $\Lambda_{i}$ as:

$$
f_{i}=\frac{1}{2 \pi} \sqrt{\frac{\Lambda_{i}}{m}}, i=1,2,3
$$

Based on the design of the flexible elements and stiffness matrices for the systems (Equation 19) we get:

$$
K=\left[\begin{array}{ccc}
0.0759 & 0 & 0 \\
0 & 0.0759 & 0 \\
0 & 0 & 0.1136
\end{array}\right] . N / \mathrm{mm}
$$

The modal directions are:
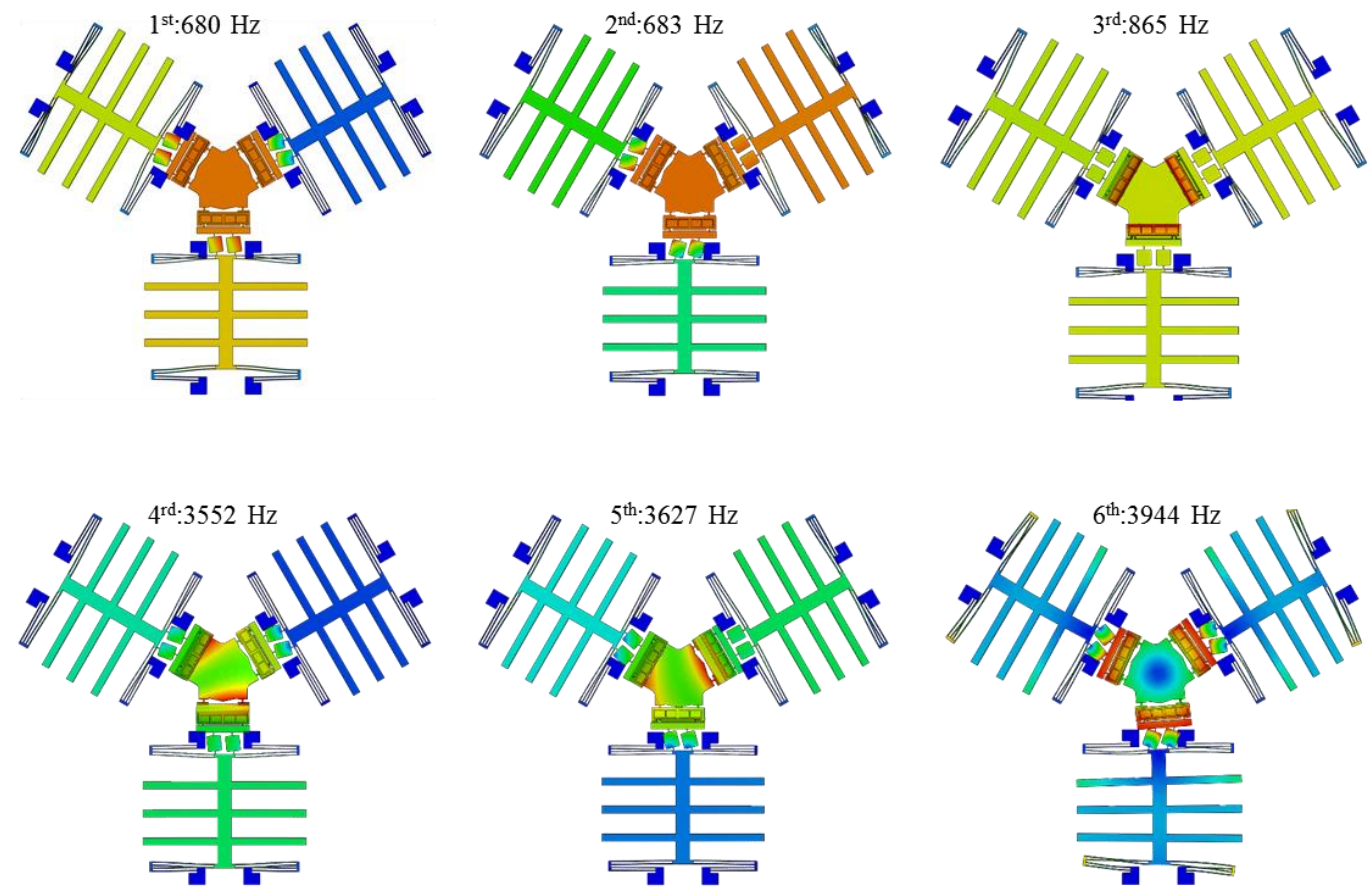

Fig. 8. Mode shapes and their corresponding frequencies (without damping). 


$$
\left[\begin{array}{l}
x_{1} \\
y_{1} \\
0
\end{array}\right],\left[\begin{array}{l}
x_{2} \\
y_{2} \\
0
\end{array}\right],\left[\begin{array}{l}
0 \\
0 \\
1
\end{array}\right]
$$

and, with a moving mass of $3.655 \times 10^{-3} \mathrm{mg}$, the frequencies for each modal directions are

$$
\left[\begin{array}{l}
630.92 \\
630.92 \\
771.75
\end{array}\right] \mathrm{Hz}
$$

Similar to reference [30], the modal directions and the diagonal structure of $K$ result from the symmetry of the structure in the XY plane. Thus, theoretically any set of orthogonal directions in the plane are modal directions for vibrations. Also, because the out-of plane structure for each kinematic chain is the same, The $\mathrm{Z}$ direction becomes an eigenvector for the stage. In the real system, due to fabrication errors, any mismatch of the stiffness of the three chains will result in the experimental observation of two very close modal frequencies corresponding to the in-plane modes.

To verify the previous results, a finite element analysis (FEA) is carried to estimate the natural frequencies and mode shapes of the system. A 3D model is generated in AutoCAD and processed in Abaqus CAE ${ }^{2}$. To reduce the number of degrees-offreedom and memory consumption, the truss-like structure of the moving parts are modeled as solid geometry and the extra mass introduced into the model is compensated for by reducing the material density to match the original mass. Due to the free standing nature of our system, it is necessary to verify the self-sagging of the stage through FEA. Sagging can lead to misalignment of the comb drives, additional stresses on the hinges and loss of motion in the manipulator. The simulation under the gravitational load showed a $52 \mathrm{~nm}$ deflection at the end effector. Figure 8 shows the first six modes of the manipulator. The first three modes correspond to vibrations along the translational degrees-of-freedom while the last three modes are related to parasitic rotations of the end-effector. Mode 1 and 2 correspond to the in-plane degrees-of-freedom of the manipulator. In mode 1 , two of the actuators move in anti-phase while the third one stays stationary. In mode 2, two of the actuators move in phase and the third actuator moves in anti-phase with twice the amplitude. Mode 3 corresponds to the translation of the table along the $Z$ direction and, in this mode, all of the actuators oscillate in phase with the same amplitude. Also shown in Figure 8 are modes 4-6 that correspond to parasitic motions. It is also interesting to note that the least stiff parasitic mode is about 17 times stiffer that the stiffest desired compliance. The dimensional parameters of the stage (e.g. $L_{1}, L_{2}$, and $\alpha_{0}$ ) and the parameters of flexible elements obtained in this section are used in final design of the PK-XYZ-MEMS stage, whose fabrication, characterization and control is reported in the second paper [31] of this set.

\section{Conclusion}

This paper has presented a detailed study and developed a design methodology for a parallel kinematics flexure-based SOIMEMS stage with three translational DOF. This scheme is an adaptation to the parallel kinematics scheme presented in [26], to make implementation as a MEMS device feasible. The direct kinematics of the device is reduced to a six order polynomial equation. However, the solution of the inverse kinematics is simple and it is use throughout this paper to study the behavior of the manipulator. Expressions for the Jacobian matrices near the nominal configuration, relating end-effector position to deflection at different flexure elements, are calculated and insight into the behavior of the mechanism is drawn from the linearized version of the kinematics. Finally, the static and dynamic behavior of the manipulator was characterized using the Lagrange's equation, and a closed form expression of the overall stiffness of the manipulator near the nominal configuration was developed.

The preceding analysis, along with designs of the individual components of the manipulator were integrated into a design approach for PK-XYZ-MEMS stages, demonstrating how the stiffness relations, constraints posed by the flexible elements and the actuators can be used determine key parameters and dimensions of a stage to meet different force amplification and spatial displacement requirements. The workspace of the stage is assessed from the kinematics equations and displacement constraints on the joints. The modes and model frequencies of the stage where calculated and verified using FEA simulations. A companion paper [31] discusses the fabrication, characterization and control of the stage.

\section{Acknowledgment}

This work was supported in part by the National Science Foundation under grants CMMI-0749028 and 0800863 . Funds from the Tungchao Julia Lu Professorship in the Department of Mechanical Science and Engineering also made this work possible. The authors acknowledge the help of Nick Toombs in preparing the 3-D renderings of the design.

\section{REFERENCES}

[1] J. Li, Q. X. Zhang and L. A. Q, "Advanced fiber optical switches using deep RIE (DRIE) fabrication," Sensors Actuators A 102, pp. 286-295, 2003.

\footnotetext{
${ }^{2}$ Abaqus/CAE by $@$ Dassault Systèmes
} 
[2] Y. Sun, B. J. Nelson, D. P. Potasek and E. A. Enikov, "Bulk microfabricated multi-axis capacitive cellular force sensor using transverse comb drives," J. Micromech. Microeng. 12 832-40, p. 12 832-40, 2002.

[3] B. Koo and P. M. Ferreira, "An active MEMS probe for fine position and force measurements," Precision Engineering, vol. 38, p. 738-748, 2014.

[4] Y. Sun, D. Piyabongkarn, A. Sezen, B. J. Nelson and R. Rajamani, "A high-aspect-ratio two-axis electrostatic microactuator with extended travel range," Sensors Actuators A 102 49-60, p. 49-60, 2002.

[5] P. F. Indermuehle, C. Linder, J. Brugger, V. P. Jaecklin and N. F. de Rooij, "Design and fabrication of an overhanging xymicroactuator with integrated tip for scanning surface profiling.," Sensors Actuators A 43, p. 346-50, 1994.

[6] P.-F. Indermuhle, V. P. Jaecklin, J. Brugger, C. Linder, N. F. de Rooij and M. Binggeli, "AFM imaging with an xymicropositioner with integrated tip. Sensors Actuators A 47," Sensors Actuators A 47, p. 562-65, 1995.

[7] M. Epitaux, J.-M. Verdeil, Y. Petremand, W. Noell and N. De Rooij, "Micro-machined XY stage for fiber optics module alignment.," IEEE Conf. on Optical Fiber Communication (Anaheim, CA), p. pp 131-3, 2005.

[8] C.-H. Kim and Y.-K. Kim, "Integration of a micro lens on a micro XY-stage.," Proc. SPIE-Int. Soc. Opt. Eng. 3892, p. 109-17, 1999.

[9] T. Kazuhiro, N. K. Ho, S. Kunihiko, M. Makoto, F. Hiroyuki and T. Hirosh, "A two-dimensional - $\theta$ micro optical lens scanner with electrostatic comb-drive XY-stage.," IEICE Electron.Express 2, p. 542-47, 2005.

[10] T. Kazuhiro, M. Makoto, F. Hiroyuki and T. Hiroshi, "A high fill-factor comb-driven XY-stage with topological layer switch architecture.," IEICE Electron. Express 3, p. 197-202, 2006.

[11] Q. Yao, P. M. Ferreira and D. Mukhopadhyay, "D Development of a novel piezo-driven parallel-kinematics single crystal silicon micropositioning XY stage.," Proc. SPIE—Int. Soc. Opt. Eng. 5836, p. 56-66, 2005.

[12] L. Yigui, S. Minoru and H. Kazihiro, "A two-dimensional self-aligning system driven by shape memory alloy actuators.," Opt. Laser Technol. 37, p. 147-9, 2005.

[13] C.-T. Wu and W. Hsu, "An electro-thermally driven microactuator with two dimensional motion," Microsyst. Technol. 8, p. 47-50, 2002.

[14] V. P. Jaecklin, C. Linder, N. F. de Rooij, M. J. M, R. Bischof and F. Rudolf, "Novel polysilicon comb actuators for xystages," Proc IEEE Micro Electro Mech Syst Workshop, p. 147-9, 1992.

[15] C. S. B. Lee, S. Han and N. C. MacDonald, "Single crystal silicon (SCS) XY-stage fabricated by DRIE and IR alignment.," Proc. IEEE Micro Electro Mechanical Systems (MEMS), p. 28-33, 2000.

[16] C.-H. Kim and Y.-K. Kim, "Micro XY-stage using silicon on a glass substrate.," J. Micromech. Microeng. 12, p. 103-7, 2002.

[17] T. Harness and R. Syms, "A Characteristic modes of electrostatic comb-drive X-Y microactuators.," J. Micromech. Microeng. 10 7-14, p. 7-14, 2000.

[18] I. Bonev, "Delta parallel robot.," [Online]. Available: http://www.parallemic.org/Reviews/Review002.html.

[19] T. Kazuhiro, "Switched-Layer Design for SOI Bulk Micromachined XYZ Stage Using Stiction Bar for Interlayer Electrical Connection.," Journal of microelectromechanical systems, pp. Vol. 18, no. 4, August 2009..

[20] X. Liu, "A MEMS stage for 3-axis nanopositioning," J. Micromech. Microeng. , p. 17 1796-1802, 2007.

[21] Y. Ando, "Development of three-dimensional electrostatic stages for scanning probe microscope," Sensors and Actuators A 114, p. 285-291, 2004.

[22] H. Dooyoung, "A self-aligned vertical comb-drive actuator on an SOI wafer for a 2D scanning micromirror," J. Micromech. Microeng., p. 14 1148-1156, 2004.

[23] Y.-S. Kim, N. G. Dagalakis and S. K. Gupta, "Design of MEMS based three-axis motion stage by incorporating a nested structure," J. Micromech. Microeng, p. 24, 2014.

[24] J.Dong and P. M. Ferreira, "Electrostatically Actuated Cantilever With SOI-MEMS Parallel Kinematic XY Stage," Journal of Microelectromechanical Systems, vol. 18, pp. 641-651, 2009.

[25] J. Kimberly A, L. Craig P and L. Howell, "An XYZ Micromanipulator with three translational degrees of freedom," Robotica, pp. 24 305-314, 2006.

[26] J.Dong and P. M. Ferreira, "A novel parallel-kinematics mechanisms for integrated, multi-axis nanopositioning Part 1. Kinematics and design for fabrication," Precision Engineering 32 (2008) 7-19, p. 32 7-19, 2008.

[27] B. Siciliano and O. Khatib, "Springer handbook of robotics.," Springer, 2008.

[28] B. Koo and P. M. Fereira, "A 2 Degree-of-Freedom SOI-MEMS Translation Stage With Closed-Loop Positioning," Journal of Microelectromechanical Systems, vol. 21, no. 1, pp. 13-22, 2012.

[29] L. Howel, Compliant Mechanisms, New York: Wiley, 2001. 
[30] R. Legtenber, A. W. Groeneveld and M. Elwenspoek, "Comb-drive actuators for large displacements," J. Micromech. Microeng, no. 6, pp. 320-329, 1996.

[31] J.Dong and P. M. Ferreira, "A novel parallel-kinematics mechanism for integrated, multi-axis nanopositioning Part 2: Dynamics, control and performance analysis.," Precision Engineering 32 (2008) 20-33, p. 32 20-33, 2008.

[32] B. Koo, J. Correa and P. M. Ferreira, "Parallel-kinematics XYZ MEMS Part. 2 Fabrication and Experimental Characterization," Precision Engineering. 


\section{Appendix A}

The polynomial equation representating the forward position kinematics of the manipulator, with design parameters of of $r=$ $2.5 \mathrm{~mm}, L_{1}=1, L_{2}=0.7071$, is given by:

Where

$$
c_{0}+c_{1} \delta+c_{2} \delta^{2}+c_{3} \delta^{3}+c_{4} \delta^{4}+c_{5} \delta^{5}+c_{6} \delta^{6}=0
$$

$$
\begin{aligned}
& c_{6}=432 \\
& c_{5}=-432.0\left(16.24-2.0 \lambda_{1}-2.0 \lambda_{2}-2.0 \lambda_{3}\right) \\
& C_{4}=-72.0\left(-653.6-10.0 \lambda_{1}^{2}-10.0 \lambda_{2}^{2}+10.0 \lambda_{2}\left(16.24-2.0 \lambda_{3}\right)+10.0 \lambda_{1}\left(16.24-2.0 \lambda_{2}-2.0 \lambda_{3}\right)+162.4 \lambda_{3}-10.0 \lambda_{3}^{2}\right) \\
& C_{3}=-144.0 \delta^{3}\left(-2.000 \lambda_{1}^{3}+\left(-7.000 \lambda_{2}-7.000 \lambda_{3}+54.14\right) \lambda_{1}^{2}+\left(-7.000 \lambda_{2}^{2}+4.000\left(27.07-3.000 \lambda_{3}\right) \lambda_{2}\right.\right. \\
& \left.-7.000 \lambda_{3}^{2}+108.3 \lambda_{3}-435.7 \lambda_{1}\right)-2.000 \lambda_{2}^{3}-2.000 \lambda_{3}^{3}+54.14 \lambda_{3}^{2}-435.7 \lambda_{3}+\lambda_{2}^{2}\left(54.14-7.000 \lambda_{3}\right)+ \\
& \left.\lambda_{2}\left(-7.000 \lambda_{3}^{2}+108.3 \lambda_{3}-435.7\right)+1158 .\right) \\
& c_{2}=-12.00\left(-4.000 \lambda_{1}^{4}+4.000\left(-7.000 \lambda_{2}-7.000 \lambda_{3}+48.73\right) \lambda_{1}^{3}+2.000\left(-24.00 \lambda_{2}^{2}+9.000\left(37.90-4.000 \lambda_{3}\right) \lambda_{2}\right.\right. \\
& \left.-24.00 \lambda_{3}^{2}+341.1 \lambda_{3}-1311 .\right) \lambda_{1}^{2}+2.000\left(-14.00 \lambda_{2}^{3}+9.000\left(37.90-4.000 \lambda_{3}\right) \lambda_{2}^{2}+4.000\left(-9.000 \lambda_{3}^{2}+146.2 \lambda_{3}-652.6\right) \lambda_{2}\right. \\
& \left.-14.00 \lambda_{3}^{3}+341.1 \lambda_{3}^{2}-2610 . \lambda_{3}+6947 .\right) \lambda_{1}-4.000 \lambda_{2}^{4}-4.000 \lambda_{3}^{4}+194.9 \lambda_{3}^{3}-2622 . \lambda_{3}^{2}+13890 . \lambda_{3}+4.000 \lambda_{2}^{3}\left(48.73-7.000 \lambda_{3}\right)+ \\
& \left.2.000 \lambda_{2}^{2}\left(-24.00 \lambda_{3}^{2}+341.1 \lambda_{3}-1311 .\right)+2.000 \lambda_{2}\left(-14.00 \lambda_{3}^{3}+341.1 \lambda_{3}^{2}-2610 . \lambda_{3}+6947 .\right)-27420\right) \\
& C_{5}=-12.00\left(4.000\left(-1.000 \lambda_{2}-1.000 \lambda_{3}+5.414\right) \lambda_{1}^{4}+4.000\left(-3.000 \lambda_{2}^{2}+\left(37.90-4.000 \lambda_{3}\right) \lambda_{2}-3.000 \lambda_{3}^{2}+37.90 \lambda_{3}-131.9\right) \lambda_{1}^{3}+\right. \\
& 21.66 \lambda_{3}^{4}-527.6 \lambda_{3}^{3}+4675 . \lambda_{3}^{2}-18280 . \lambda_{3}+4.000 \lambda_{2}^{4}\left(5.414-1.000 \lambda_{3}\right)+4.000 \lambda_{2}^{3}\left(-3.000 \lambda_{3}^{2}+37.90 \lambda_{3}-131.9\right)+ \\
& 2.000 \lambda_{2}^{2}\left(-6.000 \lambda_{3}^{3}+129.9 \lambda_{3}^{2}-915.4 \lambda_{3}+2337 .\right)+28550{ }^{*} 2.000\left(-6.000 \lambda_{2}^{3}+12.00\left(10.83-1.000 \lambda_{3}\right) \lambda_{2}^{2}\right. \\
& \left.+\left(-12.00 \lambda_{3}^{2}+194.9 \lambda_{3}-915.4\right) \lambda_{2}-6.000 \lambda_{3}^{3}+129.9 \lambda_{3}^{2}-915.4 \lambda_{3}+2337 .\right) \lambda_{1}^{2} \\
& +2.000\left(-2.000 \lambda_{2}^{4}+2.000\left(37.90-4.000 \lambda_{3}\right) \lambda_{2}^{3}+\left(-12.00 \lambda_{3}^{2}+194.9 \lambda_{3}-915.4\right) \lambda_{2}^{2}\right. \\
& \left.+4.000\left(-2.000 \lambda_{3}^{3}+48.73 \lambda_{3}^{2}-389.7 \lambda_{3}+1152 .\right) \lambda_{2}-2.000 \lambda_{3}^{4}+75.80 \lambda_{3}^{3}-915.4 \lambda_{3}^{2}+4610 . \lambda_{3}-9139 .\right) \lambda_{1}+ \\
& \left.2.000 \lambda_{2}\left(-6.000 \lambda_{2}^{3}+12.00\left(10.83-1.000 \lambda_{3}\right) \lambda_{2}^{2}+\left(-12.00 \lambda_{3}^{2}+194.9 \lambda_{3}-915.4\right) \lambda_{2}-6.000 \lambda_{3}^{3}+129.9 \lambda_{3}^{2}-915.4 \lambda_{3}+2337 .\right)\right) \\
& C_{6}=\left(4.000\left(-2.000 \lambda_{2}^{2}+\left(16.24-2.000 \lambda_{3}\right) \lambda_{2}-2.000 \lambda_{3}^{2}+16.24 \lambda_{3}-43.97\right) \lambda_{1}^{4}+4.000 \lambda_{2}^{4}\left(-2.000 \lambda_{3}^{2}+16.24 \lambda_{3}-43.97\right)+\right. \\
& 4.000 \lambda_{2}^{3}\left(-2.000 \lambda_{3}^{3}+48.73 \lambda_{3}^{2}-307.8 \lambda_{3}+714.2\right)+6.000 \lambda_{2}\left(10.83 \lambda_{3}^{4}-205.2 \lambda_{3}^{3}+1623 . \lambda_{3}^{2}-6034 . \lambda_{3}+9516 .\right)+ \\
& 2.000 \lambda_{2}^{2}\left(-4.000 \lambda_{3}^{4}+97.46 \lambda_{3}^{3}-1043 . \lambda_{3}^{2}+4869 . \lambda_{3}-9315\right. \text {. } \\
& +4.000\left(-2.000 \lambda_{2}^{3}+\left(48.73-4.000 \lambda_{3}\right) \lambda_{2}^{2}+\left(-4.000 \lambda_{3}^{2}+64.97 \lambda_{3}-307.8\right) \lambda_{2}-2.000 \lambda_{3}^{3}+48.73 \lambda_{3}^{2}-307.8 \lambda_{3}+714.2\right) \lambda_{1}^{3} \\
& +2.000\left(+2.000 \lambda_{1}\left(\left(32.49-4.000 \lambda_{3}\right) \lambda_{2}^{4}+2.000\left(-4.000 \lambda_{3}^{2}+64.97 \lambda_{3}-307.8\right) \lambda_{2}^{3}+\right.\right. \\
& \left(-8.000 \lambda_{3}^{3}+194.9 \lambda_{3}^{2}-1559 . \lambda_{3}+4869 .\right) \lambda_{2}^{2}+\left(-4.000 \lambda_{3}^{4}+129.9 \lambda_{3}^{3}-1559 . \lambda_{3}^{2}+8181 . \lambda_{3}-18100 .\right) \lambda_{2} \\
& \left.\left.+3.000\left(10.83 \lambda_{3}^{4}-205.2 \lambda_{3}^{3}+1623 . \lambda_{3}^{2}-6034 . \lambda_{3}+9516 .\right)\right)\right) \lambda_{1}^{2} \\
& -3.000\left(58.63 \lambda_{3}^{4}-952.3 \lambda_{3}^{3}+6210 . \lambda_{3}^{2}-19030 . \lambda_{3}+24470 .\right)+2.000 \lambda_{1}\left(\left(32.49-4.000 \lambda_{3}\right) \lambda_{2}^{4}\right. \\
& +2.000\left(-4.000 \lambda_{3}^{2}+64.97 \lambda_{3}-307.8\right) \lambda_{2}^{3}+\left(-8.000 \lambda_{3}^{3}+194.9 \lambda_{3}^{2}-1559 . \lambda_{3}+4869 .\right) \lambda_{2}^{2}+ \\
& \left(-4.000 \lambda_{3}^{4}+129.9 \lambda_{3}^{3}-1559 . \lambda_{3}^{2}+8181 . \lambda_{3}-18100 .\right) \lambda_{2}+3.000\left(+2.000 \lambda_{1}\left(\left(32.49-4.000 \lambda_{3}\right) \lambda_{2}^{4}\right.\right. \\
& +2.000\left(-4.000 \lambda_{3}^{2}+64.97 \lambda_{3}-307.8\right) \lambda_{2}^{3}+\left(-8.000 \lambda_{3}^{3}+194.9 \lambda_{3}^{2}-1559 . \lambda_{3}+4869 .\right) \lambda_{2}^{2}+ \\
& \left.\left.\left.\left(-4.000 \lambda_{3}^{4}+129.9 \lambda_{3}^{3}-1559 . \lambda_{3}^{2}+8181 . \lambda_{3}-18100 .\right) \lambda_{2}+3.000\left(10.83 \lambda_{3}^{4}-205.2 \lambda_{3}^{3}+1623 . \lambda_{3}^{2}-6034 . \lambda_{3}+9516 .\right)\right)\right)\right)
\end{aligned}
$$




\section{Appendix B}

Parametric finite element simulations show the computed angular errors for different positions of the table as it moves along edges of the boundary of the workspace. Figure B (a) shows the path of actuation while figure B (b) shows the recorded values of angular errors. The maximum allowed displacements for the comb drives is $25 \mu \mathrm{m}$ and the maximum titling error observed is 2.6 milli-radians. Other paths along the edges of the workspace to the point of maximum z-displacement are possible but, because of symmetry of the workspace (and the structure) around the z-axis, are equivalent to the path taken. Analysis of the causes of these errors indicates that they accrue from the bending of the folded leaf springs, which can be reduced by increasing the thickness of device layer DL2.
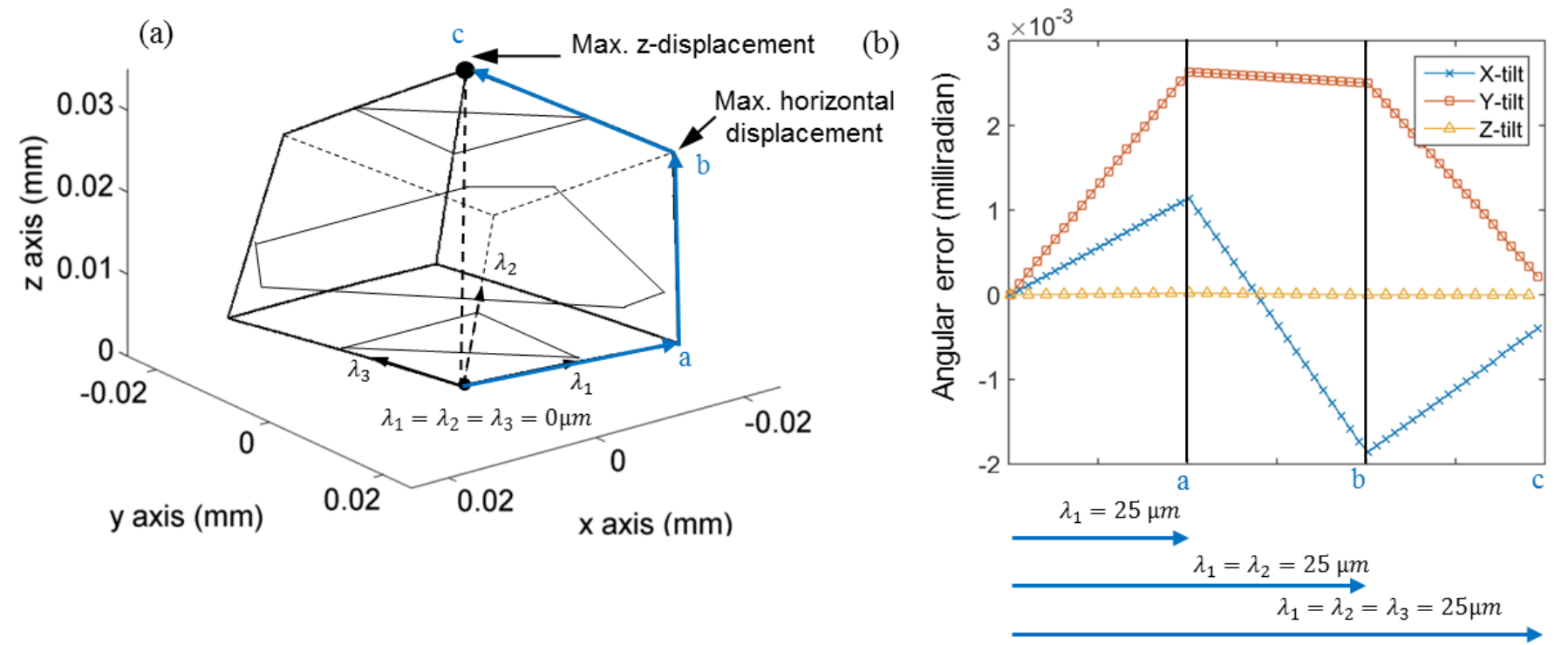

Fig. B. (a) Path of actuation at the moment of computing the angular parasitic errors and (b) Evolution of the angular errors 


\title{
Graphical Abstract
}

For

\section{Parallel-kinematics XYZ MEMS Part 1: Kinematics and Design for Fabrication}

\author{
Jorge Correa, Bonjin Koo, and Placid Ferreira*
}

Department of Mechanical Science and Engineering, 1206 West Green Street, MC-244, University of Illinois at Urbana-Champaign, Urbana, IL 61801, USA

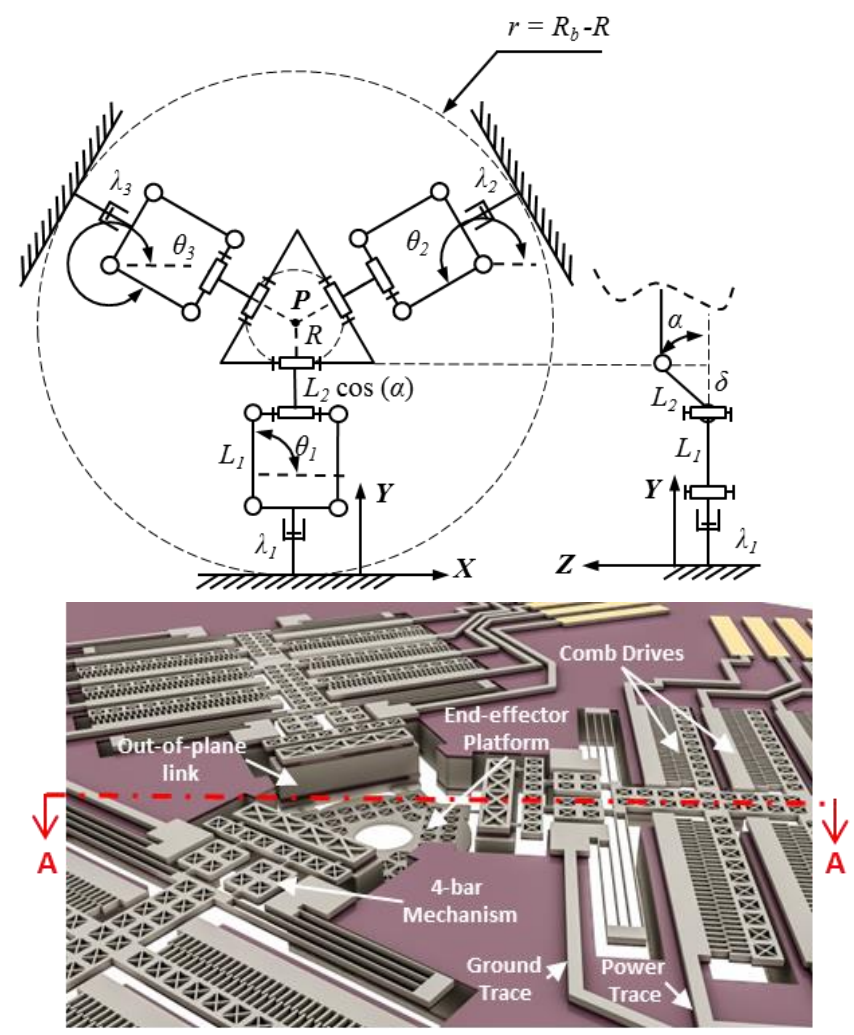

Section AA

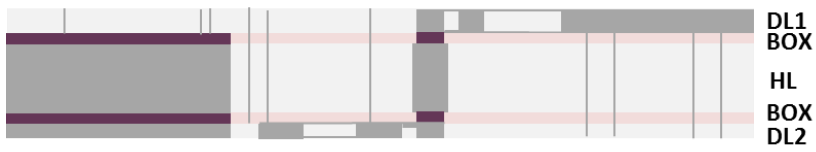

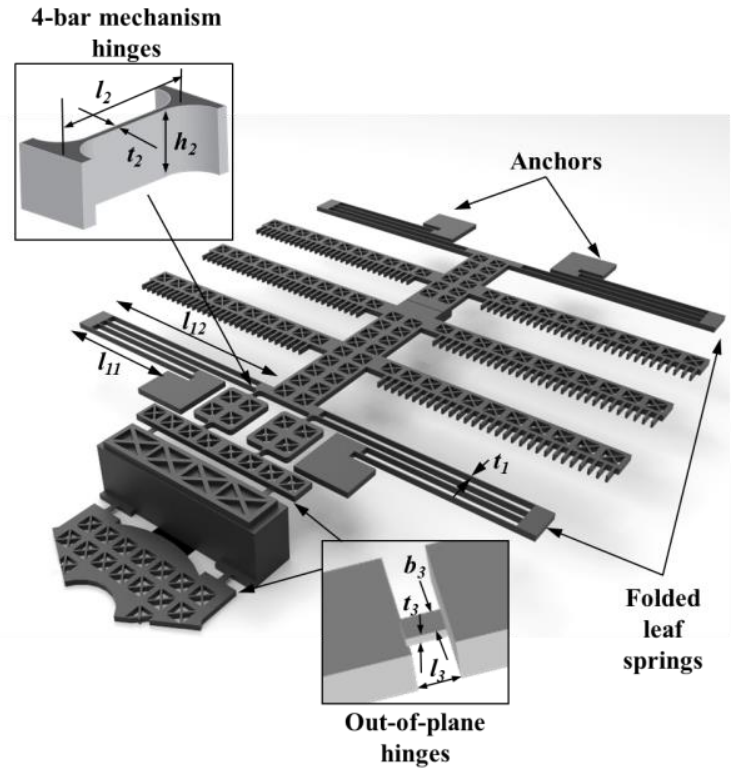

DL1

$\mathrm{HL}$

DL2

A 3-dimensional Parallel-kinematics XYZ Micro-Electro-Mechanical Positioning System: (Top left) Schematic of PK-XYZ-MEMS kinematics showing the important global parameters and variables. (Bottom left) 3-D rendering of stage layout showing important elements. Different layers (Device Layers 1 and 2, Handle, and BOX layers) of the substrate are visible in section. (Right) Solid model of a single kinematic chain with flexure joints and important parameters defining them. 